\title{
Structure of a new bulk Ti5Al2C3 MAX phase produced by the topotactic transformation of Ti2AlC
}

\author{
Nina J. Lane, Michael Naguib, Jun Lu, Lars Hultman and Michel W. Barsoum
}

\section{Linköping University Post Print}

N.B.: When citing this work, cite the original article.

Original Publication:

Nina J. Lane, Michael Naguib, Jun Lu, Lars Hultman and Michel W. Barsoum, Structure of a new bulk Ti5Al2C3 MAX phase produced by the topotactic transformation of Ti2AlC, 2012, Journal of the European Ceramic Society, (32), 12, 3485-3491.

http://dx.doi.org/10.1016/j.jeurceramsoc.2012.03.035

Copyright: Elsevier http://www.elsevier.com/

Postprint available at: Linköping University Electronic Press

http://urn.kb.se/resolve?urn=urn:nbn:se:liu:diva-79646 
Structure of bulk Ti5Al2C3 produced by the topotactic transformation of Ti2AIC

Nina J. Lane,1,* Michael Naguib,1,2 Jun Lu,3 Lars Hultman,3 and Michel W. Barsoum

1Department of Materials Science and Engineering, Drexel University, Philadelphia, PA 19104, USA

2 A. J. Drexel Nanotechnology Institute, Drexel University, Philadelphia, PA 19104, USA

3Department of Physics, Chemistry, and Biology (IFM), Linköping University, SE-581 83 Linköping, Sweden

*Corresponding author. Email address: lane@drexel.edu

Keywords: Carbides, Sintering, Electron Microscopy, X-ray methods, Powders-solid state reaction

Abstract

Upon annealing cold-pressed Ti2AIC, -325 mesh powders, at $1500^{\circ} \mathrm{C}$ for $8 \mathrm{~h}$ in argon, the resulting partially sintered sample contained $43( \pm 2)$ wt.\% of the layered ternary carbide Ti5Al2C3. Herein, the $\mathrm{X}$-ray powder diffraction pattern of Ti5Al2C3 is reported for the first time. Its structure and stoichiometry are confirmed through high-resolution transmission electron microscopy. This phase has a trigonal structure (space group P3) with a unit cell consisting of 3 formula units and cell parameters of $a=3.064 \AA, c=48.23 \AA$. The lattice parameters determined through first principles calculations agree reasonably well with the experimentally determined values. At $147.1 \mathrm{GPa}$, the calculated bulk modulus falls between the bulk moduli of the MAX phases Ti2AIC and Ti3AIC2. The transformation from Ti2AlC to Ti5Al2C3 is topotactic.

1. Introduction

Binary, early transition metal carbides possess a number of desirable properties including hightemperature stability, high melting point, high hardness, and low compressibility relative to metals; however, their applications are limited because they are brittle, difficult to machine, and highly susceptible to thermal shock. The Ti-Al-C system includes ternary phases that possess ceramic-like properties of their binary relative, $\mathrm{TiC}$, while overcoming many shortcomings of the latter by acquiring some of the more favorable properties of metals. Most notably, two of the ternaries in the system are part of a family of nanolaminated compounds known as MAX phases, which have the general formula $M n+1 A X n(n=1-3)$ where $M$ is an early transition metal, $A$ is an element from groups IIIA or IVA, and X is C or N. They can be further characterized according to their value of $n$ : "211" for $n=1$, “312" for $n=2$, and " 413 " for $n=3$. 
The synthesis of Ti2AIC was first reported in the 1960s [1], along with other 312 phases, including Ti3SiC2 [2]. Several decades later in 1994, Pietzka and Schuster synthesized Ti3AIC2, for the first time, and found that it was isostructural with Ti3SiC2 [3]. It was later discovered that, as a class, the MAX phases have unusual yet attractive and sometimes unique combinations of properties, and these phases have since attracted a great deal of interest [4-7]. They are excellent electric and thermal conductors with exceptional thermal shock resistance. While they are elastically quite stiff, they are also relatively soft and readily machinable, with exceptional damage tolerance $[4,5,8]$. Some are creep and fatigue resistant [9-11]. Of the $>60$ MAX phases known to date, Ti2AIC and Ti3AIC2 are particularly significant when considering the transition of MAX phases from the laboratory to practical applications. They have perhaps the greatest potential for commercialization due to their excellent oxidation resistance and the accessibility of their starting materials, which are relatively inexpensive and readily available $[12,13]$.

In the Ti-Al-C system, three ternary compounds have been reported to date. Two of them are the MAX phases Ti3AIC2 and Ti2AIC, which crystallize in a hexagonal lattice (space group P63/mmc) composed of $\mathrm{M}-\mathrm{X}$ octahedra stacked between layers of the A element. Another ternary carbide, Ti3AIC, has a structure similar to oxide perovskites, with Ti and Al forming an FCC-like structure and $C$ in the body-centered octahedral hole $[14,15]$.

Since Ti2AIC and Ti3AIC2 are some of the more promising MAX phases for industrial and commercial uses, the ability to fine-tune their stoichiometry and crystal structure (for example, by altering their stacking sequences) would open more opportunities for engineering their properties. In the present study, we report on the synthesis of a new MAX phase, Ti5Al2C3. Its stacking sequence can be considered as alternate layers of Ti2AIC and Ti3AIC2. This phase has previously been reported by Lin et al in Ti2AIC bulk samples [16] and by Wilhelmsson et al in Ti2AIC thin films [17]. In both cases, however, it was found in samples that were predominately single-phase Ti2AIC. In other words, it was only observed in small domains through transmission electron microscope, TEM, analysis. Consequently, its X-ray diffraction, XRD, patterns are unknown.

Herein, we show that Ti5Al2C3 can be synthesized in bulk. We determine its long-range crystal structure, stacking, and stoichiometry through XRD and TEM analysis. We also perform first principles calculations to determine its ground state parameters and its electronic structure.

\section{Experimental Details}

The sample was made by heating Ti2AIC powders that were commercially obtained (3-ONE-2, Voorhees, NJ, > $92 \mathrm{wt}$.\% purity; particle size $<44 \mu \mathrm{m}$, i.e., -325 mesh) in an alumina tube furnace under flowing argon, $\mathrm{Ar}$, at $10^{\circ} \mathrm{C} / \mathrm{min}$ to $1500{ }^{\circ} \mathrm{C}$. The powders were then held at temperature for 8 h. After cooling to room temperature, powders were obtained from the resultant partially sintered bulk piece (relative density of $\approx 80 \%$ ) using a titanium nitride coated milling bit.

XRD patterns were obtained with a powder diffractometer (Rigaku Smartlab, Japan) using Cu Ka radiation and a step scan of $0.02^{\circ}$, with $1 \mathrm{~s}$ per step. Si powder was added to some samples as an internal standard to calibrate the diffraction angles and the instrumental peak broadening. Rietveld analysis was performed using Material Analysis by Diffraction/Reflectivity (MAUD) software [18, 19], 
assuming the presence of Ti2AIC, Ti3AIC2, $\mathrm{Si}$ (used as an internal standard) and a proposed phase Ti5Al2C3 (Fig. 3a). The phase fractions, background, lattice parameters (except for Si), thermal factors, and symmetry-constrained atomic positions were refined using the least-squares refinement implemented in MAUD.

Samples for TEM observation were collected by a holey carbon grid after suspending the powder in ethanol. The TEM used (FEI Tecnai G2 TF 20 UT) had a $0.19 \mathrm{~nm}$ point resolution and $200 \mathrm{kV}$ working voltage. The crystal structure of the new phase was investigated by selected electron diffraction (SAED) and high resolution TEM (HRTEM), together with image simulation.

$A b$ initio calculations, based on density functional theory (DFT), were performed using the PerdewBurke-Eruzerhof (PBE) generalized gradient approximation (GGA) [20] with a plane wave cutoff of $400 \mathrm{eV}$. The total energy was converged to $<0.02 \mathrm{meV} /$ atom with a $13 \times 13 \times 2 \Gamma$-centered k-point grid, as implemented in the VASP software. For Ti, the semi-core $3 p$ states were treated as valence electrons. Computations of the electronic properties and structural parameters were carried with calculations of the total density of states (DOS) and energy minimization through the relaxation of atomic positions and c/a ratio for a range of fixed volumes. The equilibrium volume and bulk moduli were obtained with the modified Morse equation of state fit of the total energy as a function of volume. [21]

\section{Results and Discussion}

The XRD pattern of the Ti2AIC powder sample before and after heat treatment is shown in Figs. 1a and $1 \mathrm{~b}$, respectively. Before heat treatment (Fig. 1a), the sample was predominantly single phase Ti2AIC (red diamond markers), with a small amount ( 9 wt.\%) of Ti3AIC2 (gray crosses). After heat treatment (Fig. 1b), additional peaks appeared corresponding to the additional phase we identified as Ti5Al2C3 (blue circles). The peaks at $2 \theta=5.60,11.0^{\circ}$ and $16.5^{\circ}$ correspond to the (0003), (0006) and (0009) peaks for Ti5Al2C3, respectively. From these distinct peaks that cannot be accounted for by Ti2AIC or Ti3AIC2, it is readily apparent that the sample contains a substantial amount of this new phase.

To emphasize and confirm the assignment of the (000l) peaks, we collected patterns from discs produced by cold pressing, CPing, the heat-treated powders at loads corresponding to a stress of $\approx$ $1 \mathrm{GPa}$. The inset of Fig. 1 shows the $2 \theta=5-20$ o region of the XRD pattern from (i) the powder sample, and (ii) the cold-pressed sample. The two patterns are normalized to the (111) peak for Si at $2 \theta=28.50$ (not shown). The (0003), (0006), and (0009) peaks of Ti5Al2C3 are labeled, and their positions are marked by blue circles. A clear increase in the intensity of these peaks and not others with CPing confirms assigning these peaks to the 000I. The MAX phases are layered and cold pressing orients the basal planes parallel to the loading direction. Note that the peak at $13.0 \mathrm{o}$ is the (0002) peak of Ti2AIC, which is also expected to increase with CPing due to preferred orientation.

Table 2 compares the XRD results obtained experimentally and calculated peaks. Out of the 320 calculated peaks, only those with relative intensities greater than 1 are shown. Rietveld refinement with MAUD gave 43( \pm 2$)$ wt.\% Ti5Al2C3 for the sample after heat treatment, with 49( \pm 2$)$ wt.\% Ti2AIC, and $7( \pm 2)$ wt. \% Ti3AlC2. The lattice parameters of Ti5Al2C3 are $a=3.064(2) \AA, c=48.23(2) \AA$ and its space group is P3 (143). 


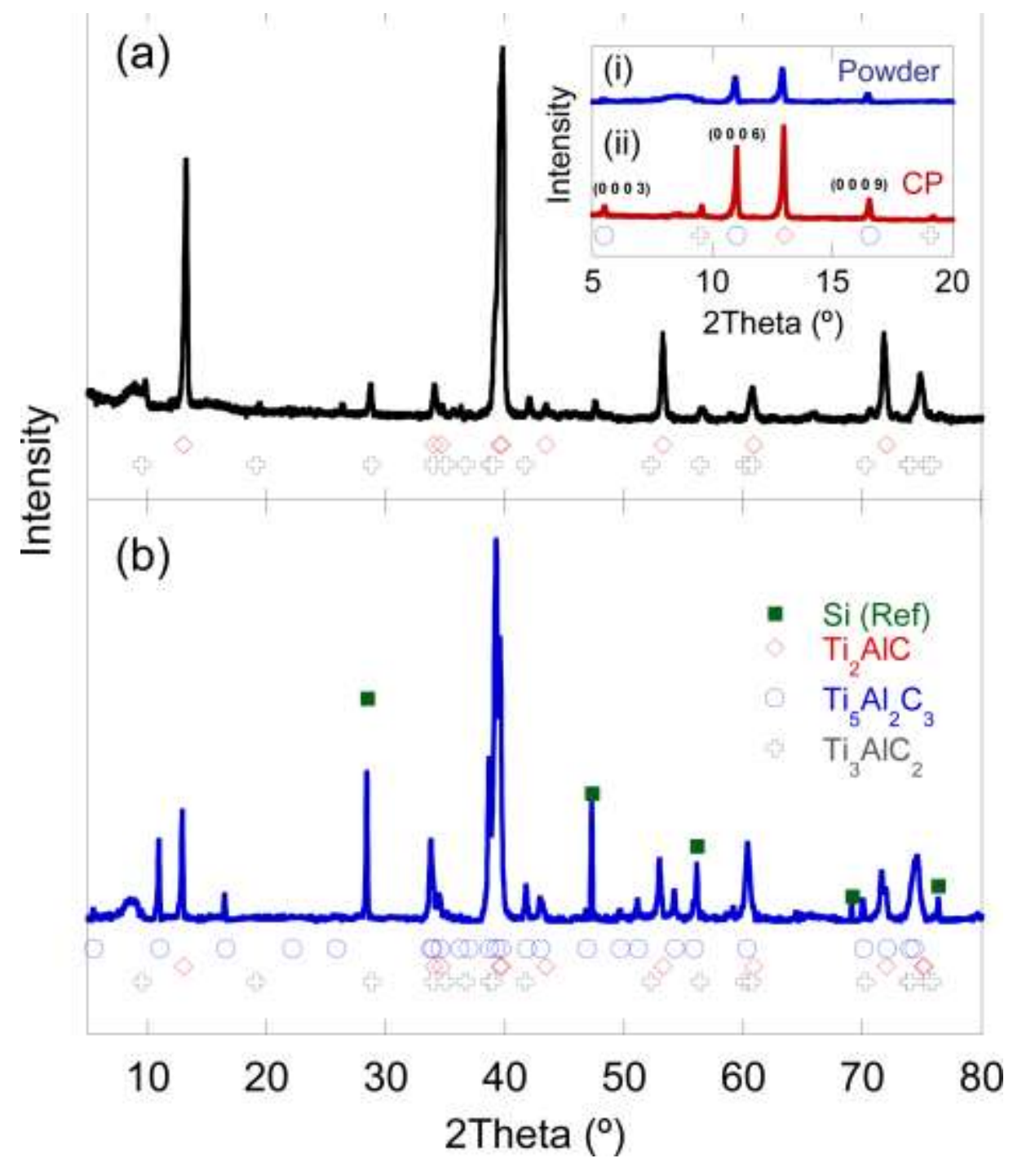

Fig. 1: XRD patterns of Ti2AIC powder sample, (a) as received and (b) after heat treatment. Inset shows region between $2 \theta=50$ and 200 for the sample after heat treatment, indexing the $\left(\begin{array}{lll}0 & 0 & 0\end{array}\right)$,

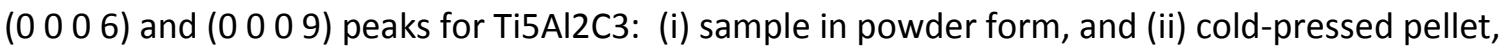
where both patterns are normalized to the Si peak at $2 \theta=28.50$ (not shown). Markers show peak positions for Si (green squares), Ti2AIC (red diamonds), Ti5AI2C3 (blue circles), and Ti3AIC2 (gray crosses). XRD patterns are shifted to accommodate markers.

The structure was confirmed by HRTEM (Fig. 2), where there are alternate layers containing 2 and 3 Ti layers sandwiched between Al layers, with C filling the octahedral sites in between Ti layers, in agreement with the simulated image of the [11 0] zone axis projection. The space group of this phase is different from those of Ti3AIC2 and Ti2AIC due to the break in symmetry caused by the alternating stacking sequence. This structure has the same stacking sequence observed in the TEM in Ref. [17].

The HRTEM image is also consistent with the equilibrium structure from first principles calculations. The full structure is shown in Fig. 3a, compared to that of Ti3AlC2 (Fig. 3b) and Ti2AIC (Fig. 3c). The stacking sequence for the Ti and $\mathrm{Al}$ atoms are shown next to the structures. A unit cell of Ti5Al2C3 consists of three formula units with a total of 6 Al layers, where every other region between the $\mathrm{Al}$ layers is filled with one Ti-C octahedra, as seen in Ti2AIC, and rest contain two Ti-C octahedra, as in 
Ti3AIC2. In order to accommodate this structure, the stacking sequence of the Al layer must shift every two layers.

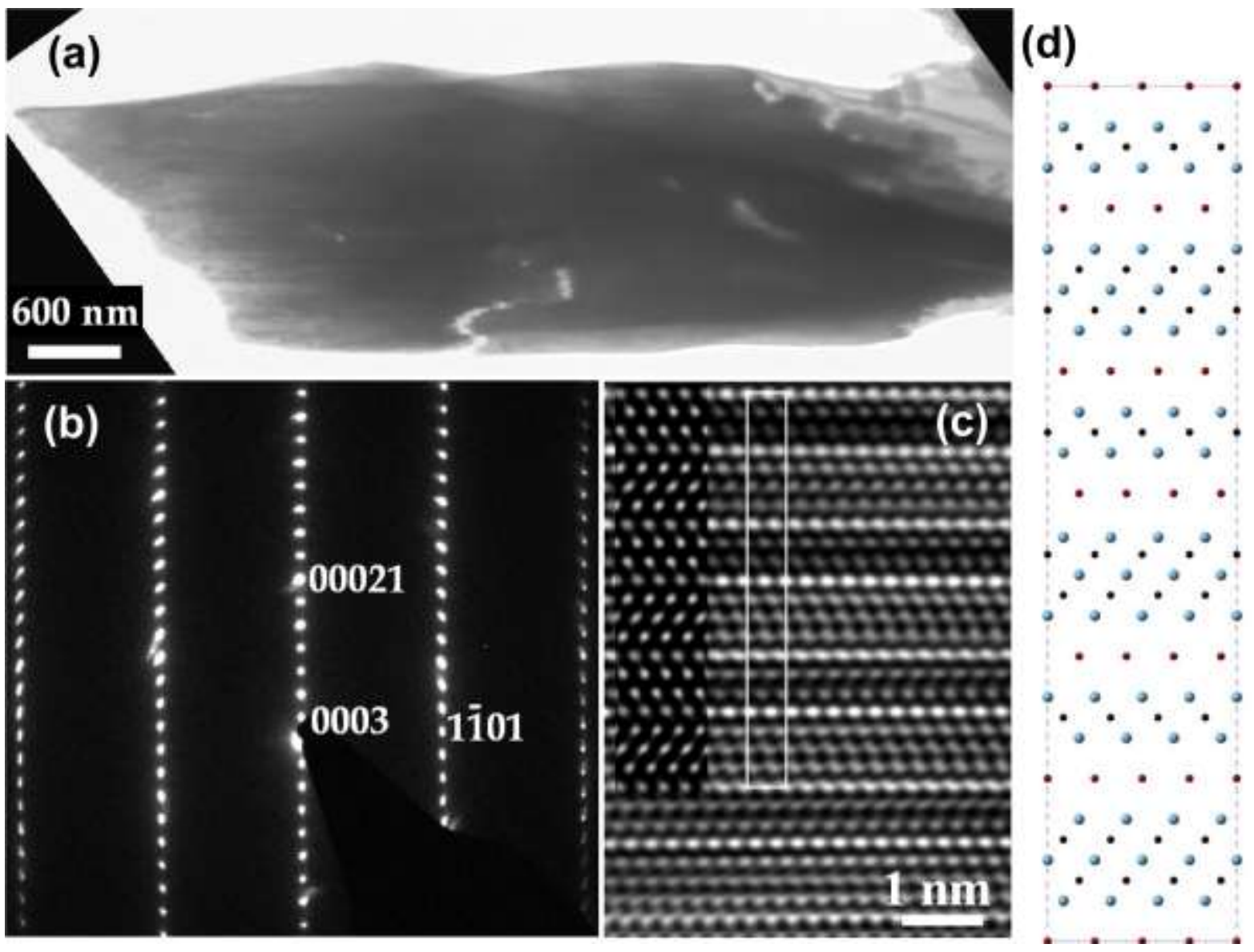

Fig. 2: High-resolution TEM (HRTEM) image of a heat-treated Ti5AI2C3 sample: (a) Large grain containing Ti5Al2C3, (b) selected area electron diffraction (SAED) pattern shown parallel to the [11 0] direction, (c) HRTEM image of the [11 0] axis projection showing Ti5AI2C3 stacking, and (d) illustration of the [11 0] plane for comparison with highlighted region in (c).

Figure $3 d$ shows the volume vs. energy plot determined by first principle calculations, as well as the equation of state fits. The bulk modulus, B0, determined through the fit is the highest for Ti3AIC2, which is apparent in Fig. 3d from its higher curvature. The bulk moduli for Ti2AIC, Ti3AIC2, and Ti5Al2C3 are listed in Table 1, where B0 varies in the order Ti2AlC $<$ Ti5Al2C3 $<$ Ti3AlC2. The lattice parameters determined by first principles calculations agree well with the experimental values from XRD and TEM (Table 1).

The calculated electronic density of states is shown in Fig. 4, where 0 corresponds to the Fermi level. The density of states is quite similar to that of Ti2AIC and Ti3AIC2 [22-24], where its conductivity is predominantly due to the Ti d orbitals. Since the stoichiometry is 2.5:1:1.5 (effectively that of a MAX phase with $n=1.5$ ), it should be expected to have electronic properties that fall somewhere between Ti2AIC and Ti3AlC2. 
$\begin{array}{lll}\text { (a) } \mathrm{Ti}_{5} \mathrm{Al}_{2} \mathrm{C}_{3} & \text { (b) } \mathrm{Ti}_{3} \mathrm{AlC}_{2} & \text { (c) } \mathrm{Ti}_{2} \mathrm{AIC}\end{array}$

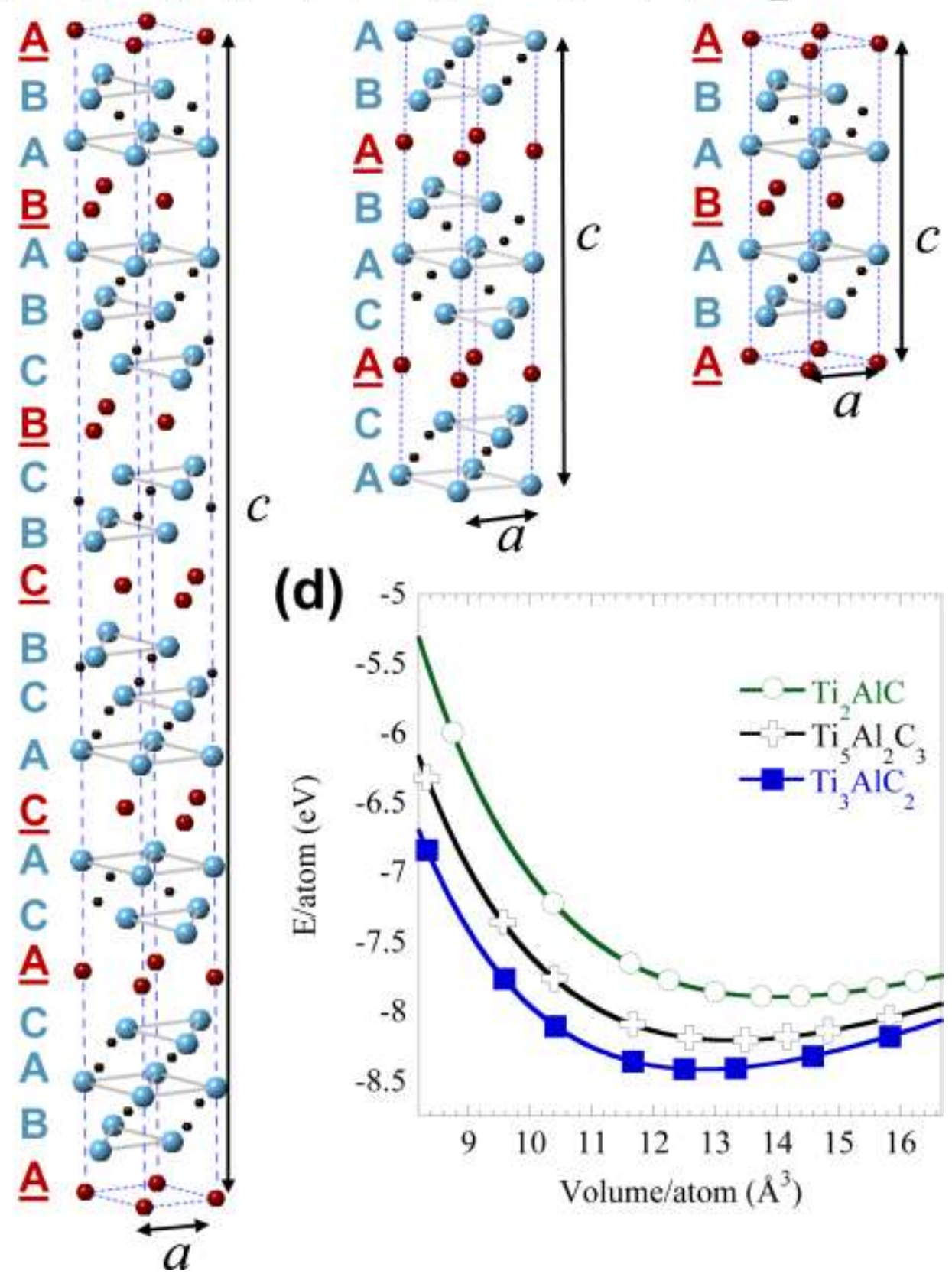

Fig. 3: Crystal structure and stacking sequence of (a) Ti5AI2C3, (b) Ti2AIC, and (c) Ti3AIC2. (d) Volume vs. energy plot for the three structures in (a)-(c), showing values determined from first principles calculations (solid data points) and the MO88 equation of state fit (lines). 
(a)

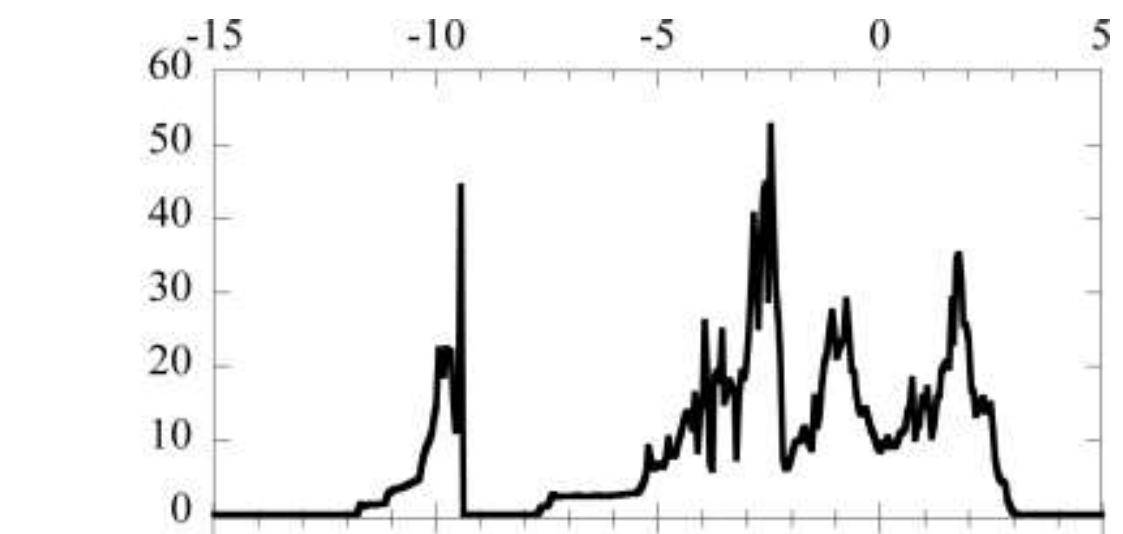

(b)

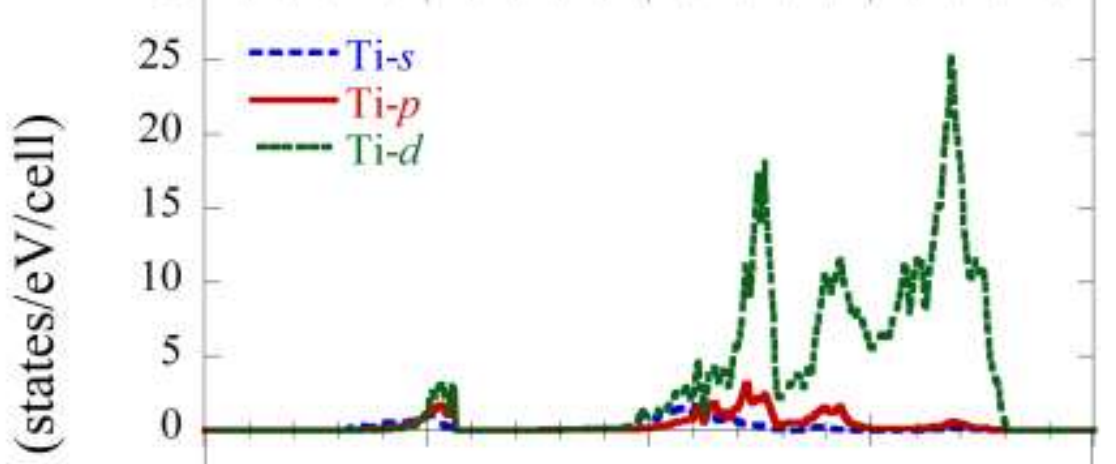

(c)

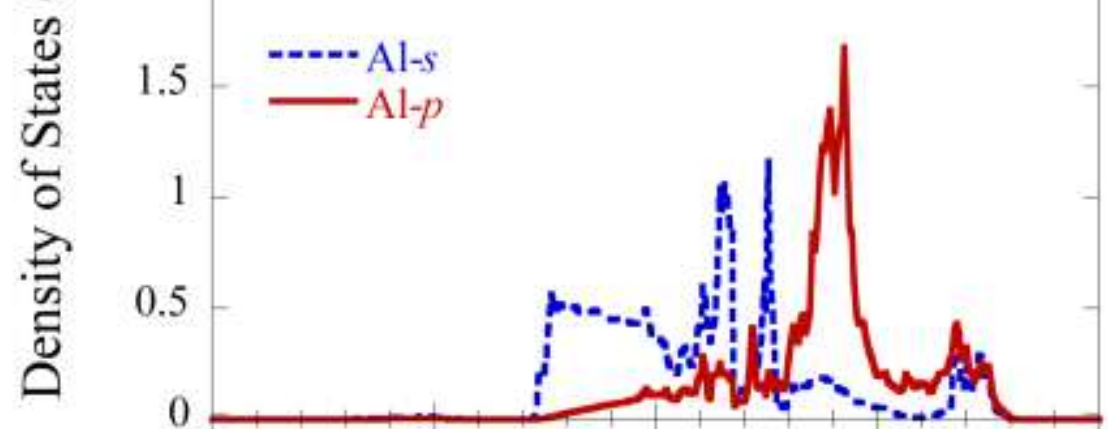

(c)

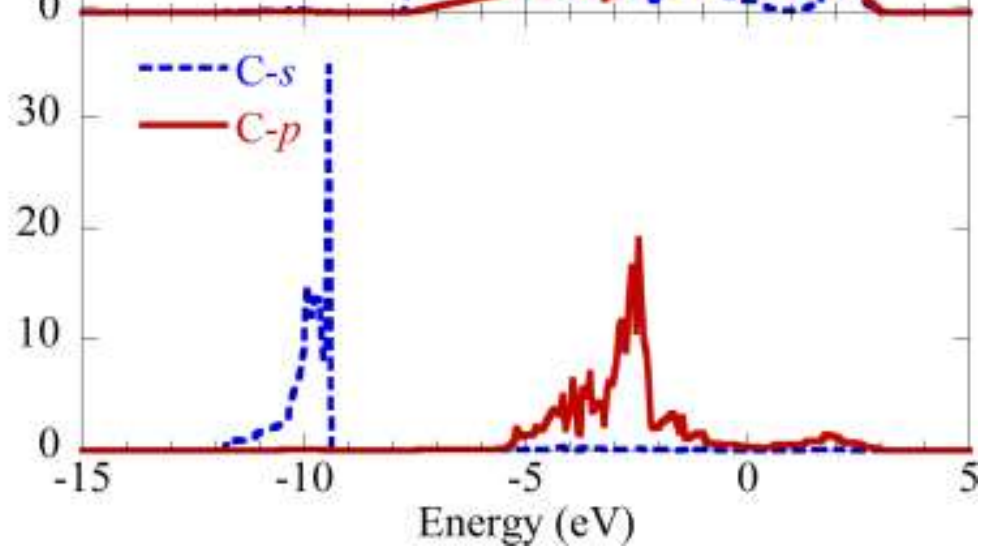

Fig. 4: Electronic density of states as determined by first principles calculations, showing (a) total density of states, and partial density of states for (b) Ti, (c) Al, and (d) C.

Since both Ti2AIC and Ti3AIC2 are generally synthesized at temperatures between 800 and 1500 oC $[13,25-28]$, the phase produced through heat treatment is highly dependent on the ratio of the starting powders. In the sample investigated in this study, we found regions where Ti5Al2C3 was present in the same grain as Ti3AlC2 (Fig. 5). It is therefore reasonable to assume that the formation 
of Ti5AI2C3 is topotactic, most likely occurring through outward diffusion of $\mathrm{Al}$ and the re-ordering of TiC octahedra in Ti2AIC. A phenomenon that could help explain the formation of Ti5Al2C3 is a socalled gradient structure, reported in a hot-pressing study of the Ti-Al-C system by Mei et al [29]. In that study, various compositions of $\mathrm{Ti}, \mathrm{Al}$, and $\mathrm{C}$ powders were hot pressed at 1500 oC for 60 minutes under $30 \mathrm{MPa}$. It was found that the samples contained an inhomogeneous structure throughout their volumes, where most of the phases were Al-deficient. Moreover, the Ti content was constant along the axial direction, whereas the Al distribution increased continuously from the hot pressed surface to the center of the sample. As a result, the relative volumes of TiC, Ti3AIC2, and Ti2AIC varied gradually. The evaporation of Al was considered a fundamental reason for such a formation, since Al has a higher vapor pressure than Ti at 1500 oC [30].
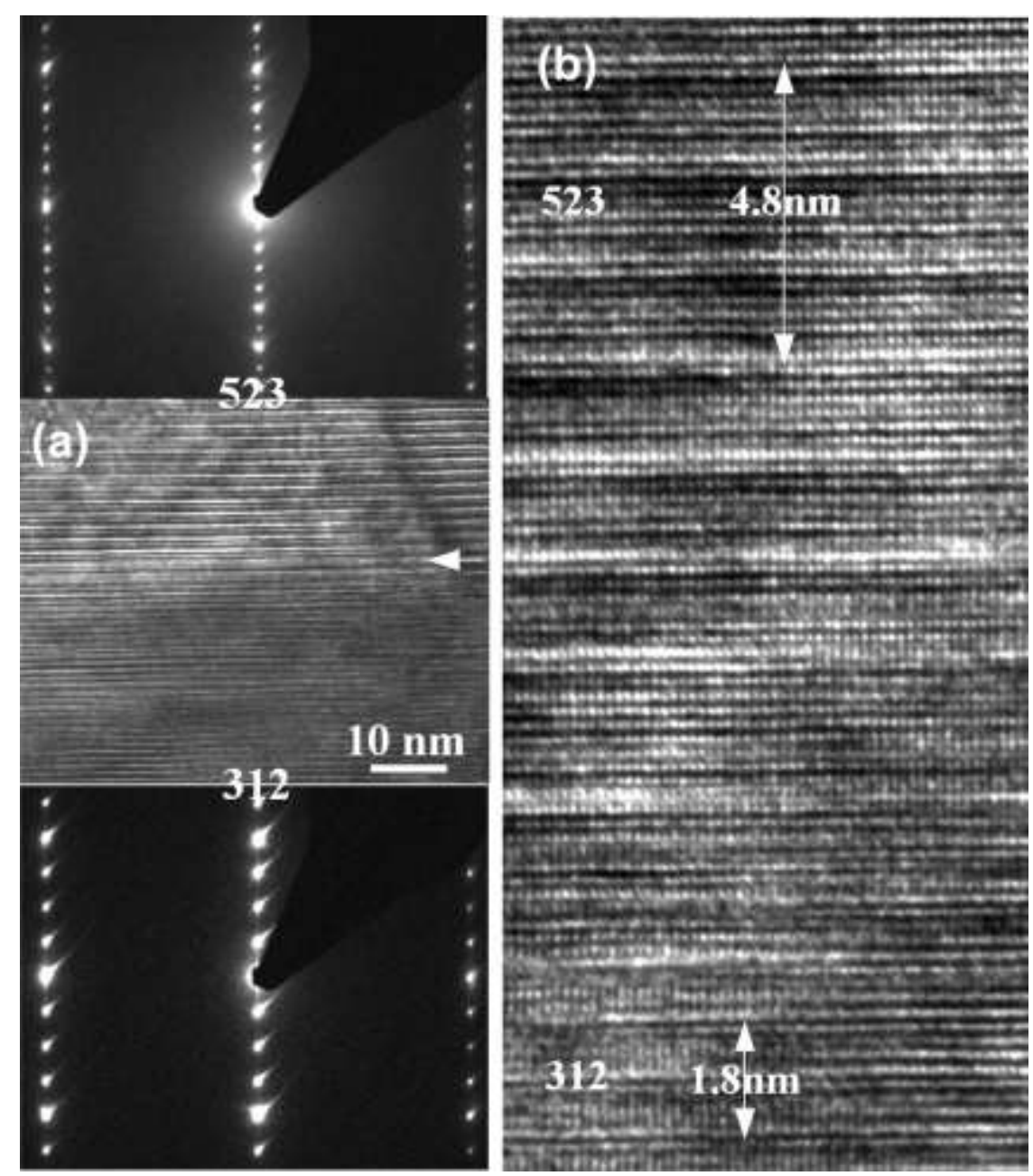

Fig. 5: HRTEM image of a grain containing both Ti5AI2C3 and Ti3AIC2 showing (a) lower-resolution and (b) higher-resolution TEM micrographs with respective unit cells indicated (the interface between the two phases is indicated by an arrow in (a)) with corresponding SAED patterns from the Ti5AI2C3 ("523") and Ti3AIC2 ("312") regions shown above and below figure (a), respectively.

Here, we postulate that the evaporation of $\mathrm{Al}$ from Ti2AlC results in a higher Ti:Al ratio, causing Ti5Al2C3 to be more thermodynamically favorable. It is reasonable to assume that further heating would convert Ti5Al2C3 to Ti3AIC2 and ultimately to TiCX. 
The results presented herein provide evidence for Ti5Al2C3 - with a stacking sequence combining that of Ti3AIC2 and Ti2AIC and properties similar to the two phases - that exists in bulk, formed when Ti2AIC powders are heated to $1500^{\circ} \mathrm{C}$ for $8 \mathrm{~h}$. Work to explore the effect of annealing times and temperatures on the microstructure and atomic ordering of ternary phases in the Ti-Al-C system would be needed to understand the kinetics and mechanisms for the formation of the Ti5Al2C3 phase.

Conclusions

A ternary phase, Ti5Al2C3, was synthesized in bulk form for the first time by heating Ti2AlC under $\mathrm{Ar}$ for $8 \mathrm{~h}$ at $1500{ }^{\circ} \mathrm{C}$. The transition from Ti2AIC to Ti5Al2C3 is topotactic. The crystal structure of Ti5Al2C3 was found to be trigonal (P3), with a and c parameters of $3.067 \AA, 48.16 \AA$, respectively. The stacking of atomic layers in Ti5Al2C3 can be considered as a combination of that in Ti2AIC and Ti3AlC2.

\section{Acknowledgements}

This work was supported by the Assistant Secretary for Energy Efficiency and Renewable Energy, Office of Vehicle Technologies of the U.S. Department of Energy under Contract No. DE-AC0205CH11231, Subcontract 6951370 under the Batteries for Advanced Transportation Technologies (BATT) Program. This work was also supported by the Commonwealth of Pennsylvania's Ben Franklin Technology Development Authority through the Ben Franklin Technology Partners of Southeastern Pennsylvania. N.L. acknowledges financial support by the Integrated Graduate Education and Research Traineeship (IGERT) under NSF grant number DGE-0654313. Use of the equipment of the Centralized Research Facility (Drexel University) is acknowledged.

References

[1] Jeitschko W, Nowotny H, Benesovsky F. Kohlenstoffhaltige ternäre Verbindungen (H-Phase). Monatsh Chem. 1963;94:672-6.

[2] Jeitschko W, H. N. Die Krystallstructur von Ti3SiC2 - Ein Neuer Komplxcarbid-Typ. Monatshefte für Chemie / Chemical Monthly. 1967;98:329-37.

[3] Pietzka MA, Schuster JC. Summary of constitution data of the system AI-C-Ti. J Phase Equilib. 1994;15:392-400.

[4] Barsoum MW, El-Raghy T. Synthesis and characterization of a remarkable ceramic: Ti3SiC2. J Am Ceram Soc. 1996;79:1953-6.

[5] El-Raghy T, Zavaliangos A, Barsoum MW, Kalidindi SR. Damage mechanisms around hardness indentations in Ti3SiC2. J Am Ceram Soc. 1997;80:513-6.

[6] Barsoum MW, El-Raghy T, Ogbuji LUJT. Oxidation of Ti3SiC2 in air. J Electrochem Soc. 1997;144:2508-16. 
[7] M.W. Barsoum TE-R. A Progress Report on Ti3SiC2, Ti3GeC2, and the H-Phases, M2BX. Journal of Materials Synthesis and Processing. 1997;5:197-216.

[8] Ganguly A, Zhen T, Barsoum MW. Synthesis and mechanical properties of Ti3GeC2 and Ti3(SixGe1-x)C2 (x=0.5, 0.75) solid solutions. J Alloy Compd. 2004;376:287-95.

[9] Radovic M, Barsoum MW, El-Raghy T, Wiederhorn S. Tensile creep of fine grained (3-5 micron) Ti3SiC2 in the 1000-1200 degrees C temperature range. Acta Mater. 2001;49:4103-12.

[10] Radovic M, Barsoum MW, El-Raghy T, Wiederhorn SM. Tensile creep of coarse-grained Ti3SiC2 in the 1000-1200 degrees C temperature range. J Alloy Compd. 2003;361:299-312.

[11] Zhen T, Barsoum MW, Kalidindi SR, Radovic M, Sun ZM, El-Raghy T. Compressive creep of fine and coarse-grained T3SiC2 in air in the 1100-1300 degrees $C$ temperature range. Acta Mater. 2005;53:4963-73.

[12] Sundberg M, Malmqvist G, Magnusson A, El-Raghy T. Alumina Forming High Temperature Silicides and Carbides. Ceramics International 2004;30:1899-904.

[13] Wang XH, Zhou YC. High-temperature oxidation behavior of Ti2AIC in air. Oxid Met. 2003;59:303-20.

[14] Riaz S, Flower HM, West DRF. Phase relationships involving TiC and Ti3AIC (P phase) in Ti-Al-C system. Mater Sci Tech Ser. 2000;16:984-92.

[15] Matar SF, Le Petitcorps Y, Etourneau J. Ab initio study of the chemical role of carbon within TiAl alloy system: Application to composite materials. Computational Materials Science. 1998;10:314-8.

[16] Lin ZJ, Zhuo MJ, Zhou YC, Li MS, Wang JY. Microstructural characterization of layered ternary Ti2AIC. Acta Mater. 2006;54:1009-15.

[17] Wilhelmsson O, Palmquist JP, Lewin E, et al. Deposition and characterization of ternary thin films within the Ti-Al-C system by DC magnetron sputtering. J Cryst Growth. 2006;291:290-300.

[18] Lutterotti L. MAUD: Material Analysis Using Diffraction. Program download, mirrored at CCP14; 1997-2009.

[19] Lutterotti L, Matthies S, Wenk H-R. MAUD: a friendly Java program for Material

Analysis Using Diffraction

Luca Lutterotti. IUCr: CPD Newsletters. 1999;21:14.

[20] Perdew JP, Burke K, Ernzerhof M. Generalized Gradient Approximation Made Simple. Phys Rev Lett. 1996;77:3865.

[21] Morse PM. Diatomic molecules according to the wave mechanics. ii. vibrational levels. Physical Review. 1929;34:57-64.

[22] Sun ZM, Music D, Ahuja R, Schneider JM. Electronic origin of shearing in $M 2 A C(M=T i, V, C r, A=$ Al, Ga). J Phys-Condens Mat. 2005;17:7169-76. 
[23] Sun ZM, Music D, Ahuja R, Li S, Schneider JM. Bonding and classification of nanolayered ternary carbides. Phys Rev B. 2004;70.

[24] Sun ZM, Ahuja R, Li S, Schneider JM. Structure and bulk modulus of M2AIC ( $M=T i, V$, and Cr). Appl Phys Lett. 2003;83:899-901.

[25] Barsoum MW, Ali M, El-Raghy T. Processing and characterization of Ti2AIC, Ti2AIN, and Ti2AIC0.5N0.5. Metall Mater Trans A. 2000;31:1857-65.

[26] Tzenov NV, Barsoum MW. Synthesis and characterization of Ti3AIC2. J Am Ceram Soc. 2000;83:825-32.

[27] Wang XH, Zhou YC. Stability and selective oxidation of aluminum in nano-laminate Ti3AIC2 upon heating in argon. Chem Mater. 2003;15:3716-20.

[28] Eklund P, Beckers M, Jansson U, Hogberg H, Hultman L. The Mn+1AXn phases: Materials science and thin-film processing. Thin Solid Films. 2010;518:1851-78.

[29] Mei BC, Hong XL, Zhu JQ, Zhou WB. Gradient structure of Ti-Al-C ternary carbide prepared by hot-pressing sintering. J Wuhan Univ Technol. 2005;20:5-7.

[30] Jacobson NS, Brady MP, Mehrotra GM. Thermodynamics of selected Ti-Al and Ti-Al-Cr alloys. Oxid Met. 1999;52:537-56.

[31] Sun Z, Ahuja R, Li S, Schneider JM. Structure and bulk modulus of M2AIC ( $M=$ Ti V and Cr). Appl Phys Lett 2003;83:899.

[32] Zhou YC, Sun Z, Wang X, Chen S. Ab initio Geometry Optimization and Ground State Properties of Layered Ternary Carbides, Ti3MC2 (M=Al Si and Ge). J Phys: Condens Matter 2001;13:10001-10.

[33] Manoun B, Zhang FX, Saxena SK, El-Raghy T, BarsoumMW. X-ray highpressure study of Ti2AIN and Ti2AIC. J Phys Chem Solids 2006;67:2091-4.

[34] Manoun B, Saxena SK. Synthesis and compressibility of Ti3(Al,Sn0.2)C2 and Ti3Al(C0.5,N0.5)2. J Appl Phys 2007;101:113523.

[35] Wang X, Zhang H, Zheng L, Ma Y, Lu X, Sun Y, Zhou Y. Ti5Al2C3: A New Ternary Carbide Belonging to MAX Phases in the Ti-Al-C System. J Am Cer Soc., 2012, http://dx.doi.org/10.1111/j.1551-2916.2012.05158.x.

[36] Palmquist J-P, Li S, Persson POÅ, Emmerlich J, Wilhelmsson O, Högberg H, Katsnelson MI, Johansson B, Ahuja R, Eriksson O, Hultman L, Jansson U. Mn+1AXn phases in the Ti-Si-C system studied by thin-film synthesis and ab initio calculations. Phys Rev B 2004;70:165401. 
Tables

Table 1 Lattice parameters of Ti5Al2C3, Ti2AIC, and Ti3AIC2 phases in powder sample determined from XRD and calculated lattice parameters, bulk modulus, and density determined from first principles calculations.

\begin{tabular}{|l|l|l|l|l|l|l|}
\hline & \multicolumn{3}{|c|}{$\mathrm{Ti}_{5} \mathrm{Al}_{2} \mathrm{C}_{3}$} & $\mathrm{Ti}_{2} \mathrm{AlC}$ & \multicolumn{2}{c|}{$\mathrm{Ti}_{3} \mathrm{AlC}_{2}$} \\
\hline & Exp. & Calc. & Exp & Calc & Exp & Calc \\
\hline$a(\AA)$ & 3.064 & 3.068 & $\begin{array}{l}3.063, \\
3.051^{\mathrm{a}}\end{array}$ & 3.067 & $\begin{array}{l}3.060, \\
3.0753^{\mathrm{d}}\end{array}$ & 3.083 \\
\hline$c(\AA)$ & 48.23 & 48.45 & $\begin{array}{l}13,645, \\
13.637^{\mathrm{a}}\end{array}$ & 13.75 & $\begin{array}{l}18.661, \\
18.578^{\mathrm{d}}\end{array}$ & 18.66 \\
\hline $\begin{array}{l}\text { Bulk } \\
\begin{array}{l}\text { Modulus } \\
(\mathrm{GPa})\end{array}\end{array}$ & ----- & 147.1 & $\left(186^{\mathrm{c}}\right)$ & $136,137^{\mathrm{b}}$ & $226^{\mathrm{f}}$ & $156,161^{\mathrm{c}}$ \\
\hline $\begin{array}{l}\text { Density } \\
\left(\mathrm{g} / \mathrm{cm}^{3}\right)\end{array}$ & ---- & 4.13 & ---- & 3.99 & & \\
\hline
\end{tabular}

aRef. [25]

bRef. [31]

cRef. [32]

dRef. [3]

eRef. [33]

fRef. [34] 